\title{
A Hybrid Model Predictive Control Approach to Attitude Control with Minimum-Impulse-Bit Thrusters*
}

\author{
Pantelis Sopasakis $^{a}$, Daniele Bernardini ${ }^{a, b}$, Hans Strauch $^{c}$, Samir Bennani $^{d}$ and Alberto Bemporad ${ }^{a, b}$
}

\begin{abstract}
This paper studies an important aspect of attitude control for a launcher's upper stage: the minimum impulse bit (MIB), that is, the minimum torque that can be exerted by the thrusters. We model this effect using principles of hybrid systems theory and we design a hybrid model predictive control scheme for the attitude control of a launcher during its long coasting period, aiming at minimizing the number of thrusters' actuations. We apply the proposed methodology to a nonlinear model of a typical upper stage with multi-payload capability.

Index Terms-Upper Stage Control, Minimum Impulse Thrusters, Hybrid Model Predictive Control, Aerospace.
\end{abstract}

\section{INTRODUCTION}

Future multi-payload launchers will have the capability to inject a payload into a geostationary transfer orbit with a payload release close to the apogee $(\mathrm{GTO}+)$. This requires the upper stage to coast up to 4 hours until the payload is jettisoned. During this long coasting period it is of high importance to achieve the pointing accuracy with a minimum amount of thruster actuations. The allowed number of total thruster firing is small especially in the case of long flight times, that are typical in GTO+ missions. Therefore, a standard high-gain tuning of the controller is not acceptable. Efficient attitude control of the upper stage of launch vehicles is of high importance for the success of their mission and must take into account several key aspects, such as keeping a low total number of actuations needed to achieve a desired level of pointing accuracy [1], [2].

Furthermore, any object in space that is exposed to solar radiation from one side and darkness on the other side is in for thermal damage due to the extreme temperatures it experiences, that can range from $125^{\circ} \mathrm{C}$ to $-50^{\circ} \mathrm{C}$ and below. As a result, the upper stage needs to rotate about its longitudinal axis at a constant rate - a maneuver known as "barbecue mode". The global objective of the control action is to track the minimizing the pitch and yaw errors caused by disturbances such as nutation and precession. Nutation and precession describe the movement of a rotating body that occurs if the rotating axis does not coincide with the axis of angular momentum [1], [3].

a IMT Institute for Advanced Studies Lucca, Piazza San Ponziano 6, 55100 Lucca, Italy. Emails: \{pantelis.sopasakis, daniele.bernardini, alberto.bemporad\}@imtlucca.it.

${ }^{b}$ ODYS Srl, via della Chiesa XXXII trav. I n. 231, 55100 Lucca, Italy.

$c$ Airbus DS, Airbus-Allee 1, 28199 Bremen, Germany. Email: hans.strauch@astrium.eads. net.

${ }^{d}$ European Space Agency (ESA), Keplerlaan 1, Noordwijk, The Netherlands, Email: samir.bennani@esa.int.

* In part, this work has been carried out in connection with ESA's Future Launcher Preparatory Program (FLPP3) study "Upper Stage Attitude Control Design Framework" (USACDF).
In the long coasting period, the upper stage is controlled by means of on/off operating thrusters. These thrusters apply a constant force on the spacecraft and they can be merely switched on and off [4]. In addition, there exists a minimum time for which the thruster can remain open, which is imposed by the characteristics of the various (mechanical and electrical) components involved in the actuation.

The presence of actuation constraints as well as the need to minimize the number of thrusters actuations motivates the choice of model predictive control (MPC) as a suitable control approach. MPC is a class of advanced control methodologies where at each sampling instant a given performance index is optimized, by taking into account the closed-loop evolution of the controlled system as predicted by a discrete-time model subject to constraints [5]. Such a model-based approach allows one to explicitly model the actuation constraints and to penalize the use of actuators in the considered performance criterion.

The use of MPC for attitude control has been proposed by Manikonda et al. [6], Vieira et al. [7] and other authors. Hegrenas et al. propose an explicit MPC control scheme for attitude control [8], [9]. Other attitude control approaches have been proposed in the literature. Xiao et al. [10] study the problem of fault-tolerant attitude control considering the saturation of the actuators. Simpler control solutions such as PD and LQR have also been proposed without, however, being able to take consistently into account the constraints that apply on the system (see [3] and references therein).

Incentivized by the switching nature of the thrusters activation mechanism, in this paper we propose a hybrid MPC scheme for attitude control of an upper stage with minimum impulse thrusters. Hybrid systems provide a unified framework for describing processes that evolve according to continuous dynamics, discrete dynamics and logic rules. Several modeling formalisms have been developed to describe hybrid systems, including piecewise affine (PWA) systems and mixed logical dynamical (MLD) systems [11], [12]. Hybrid model predictive control (which is, model predictive control based on hybrid systems) has been mainly applied to problems in the automotive domain, addressing, for example, semi-active suspension control [13], traction control [14], and active steering for vehicle stabilization and yaw regulation [15], [16], just to mention a few.

In this paper we model the minimum impulse bit using hybrid systems theory and propose a hybrid model predictive control approach aiming at minimizing the number of actuations by the thrusters. The minimization of these activations results not only in lower consumption of propellant, but also 


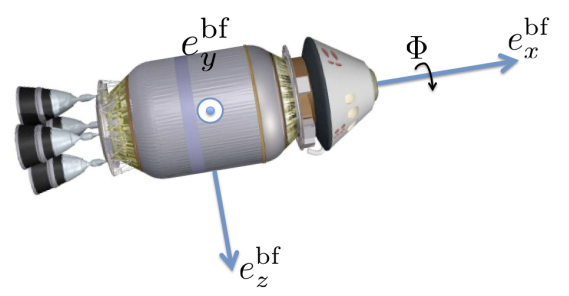

Fig. 1: Body fixed (BF) frame aligned to the principal axis of the spacecraft.

in the prolongation of the thrusters' lifetime.

Notation. Let $\mathbb{N}, \mathbb{R}, \mathbb{R}^{n}, \mathbb{R}^{m \times n}$ be the sets of natural numbers, real numbers, real $n$-vectors and real $m$-by- $n$ matrices. The set of integer numbers between $k_{1}$ and $k_{2} \geq k_{1}$ is denoted by $\mathbb{N}_{\left[k_{1}, k_{2}\right]}$. Let $P$ be a logical proposition. We denote by $[P]$ its truth value, i.e., $[P]=1$ if $P$ is true and $[P]=0$ otherwise.

\section{UPPER STAGE MODEL AND ACTUATION CONSTRAINTS}

\section{A. Upper Stage Dynamics}

In this paper we study the attitude dynamics using a body-fixed (BF) frame which is a right-handed, orthonormal reference frame fixed to the spacecraft so that the $x$-axis is aligned to its principal axis and the rotation about it is denoted by $\Phi$ and is called the roll angle. The rotational displacement about the $y$-axis defines the pitch angle $\Theta$ and the rotation about the $z$-axis is the yaw angle $\Psi$. The $\mathrm{BF}$ body frame is illustrated in Figure 1.

Let us define the state vector

$$
\chi(t) \triangleq\left[\begin{array}{lllll}
\tilde{\Theta}_{x}(t) & \tilde{\Psi}_{x}(t) & \omega_{x}(t) & \omega_{y}(t) & \omega_{z}(t)
\end{array}\right]^{\prime},
$$

where $\tilde{\Theta}_{x}(t)$ and $\tilde{\Psi}_{x}(t)$ are the pitch and yaw error angles that describe the rotational displacement of the spin axis with respect to the desired spin axis in the $x z$-plane and in the $x y$-plane, respectively. By $\omega_{x}(t), \omega_{y}(t), \omega_{z}(t)$ we denote the angular rates about the $x, y$ and $z$ axes, respectively. Let $\tau_{x}(t), \tau_{y}(t), \tau_{z}(t)$ be the torques applied by the thrusters along these axes. Then, the following continuous-time nonlinear model can be derived:

$$
\begin{aligned}
\dot{\chi}(t) & =\left[\begin{array}{ccccc}
0 & \omega_{x}(t) & 0 & 1 & 0 \\
-\omega_{x}(t) & 0 & 0 & 0 & 1 \\
0 & 0 & 0 & 0 & 0 \\
0 & 0 & 0 & 0 & \omega_{n}(t) \\
0 & 0 & 0 & -\omega_{n}(t) & 0
\end{array}\right] \chi(t) \\
& +\left[\begin{array}{c}
0_{2 \times 3} \\
J^{-1}
\end{array}\right]\left[\begin{array}{c}
\tau_{x}(t) \\
\tau_{y}(t) \\
\tau_{z}(t)
\end{array}\right]
\end{aligned}
$$

assuming that the inertia tensor can be approximated by the diagonal matrix $J^{-1}=\operatorname{diag}\left(J_{x x}^{-1}, J_{y y}^{-1}, J_{z z}^{-1}\right)$ and setting $\omega_{n}(t) \triangleq\left(1-J_{x x} / J_{y y}\right) \omega_{x}(t)$. The reader is referred to [3] for details regarding the derivation of (2).

The coupling terms $\omega_{x}(t)$ in (2) lead to nonlinearity. However, assuming that $\omega_{x}(t)$ equilibrates fast at its setpoint $\omega_{x}^{r}$, the system (2) can be approximated with a linear time-invariant system which, in fact, can be decoupled into a spin model and a nutation/precession model. The spin model describes the spin of the spacecraft independently of the pitch and yaw angles and rates, while the nutation/precession model requires no roll or roll rate information. Under the assumption $\omega_{x}(t)=\omega_{x}^{r}$, the roll rate dynamics is

$$
\dot{\omega}_{x}(t)=J_{x x}^{-1} \tau_{x}(t)
$$

and the model for nutation/precession control is

$$
\begin{aligned}
{\left[\begin{array}{c}
\dot{\tilde{\Theta}}_{x}(t) \\
\dot{\tilde{\Psi}}_{x}(t) \\
\dot{\omega}_{y}(t) \\
\dot{\omega}_{z}(t)
\end{array}\right] } & =\left[\begin{array}{cccc}
0 & \omega_{x}^{r} & 1 & 0 \\
-\omega_{x}^{r} & 0 & 0 & 1 \\
0 & 0 & 0 & \omega_{n}^{r} \\
0 & 0 & -\omega_{n}^{r} & 0
\end{array}\right]\left[\begin{array}{c}
\tilde{\Theta}_{x}(t) \\
\tilde{\Psi}_{x}(t) \\
\omega_{y}(t) \\
\omega_{z}(t)
\end{array}\right] \\
+ & {\left[\begin{array}{cc}
0 & 0 \\
0 & 0 \\
J_{y y}^{-1} & 0 \\
0 & J_{z z}^{-1}
\end{array}\right]\left[\begin{array}{c}
\tau_{y}(t) \\
\tau_{z}(t)
\end{array}\right] }
\end{aligned}
$$

where $\omega_{n}^{r} \triangleq\left(1-J_{x x} / J_{y y}\right) \omega_{x}^{r}$. Therefore, spin and nutation/precession control can be taken up by independent control modules. In particular, being (3) a single-input singleoutput system, the spin can be controlled by a simple LQR regulator. We will show in Section IV that the roll rate set-point is reached fast compared to the pitch and yaw dynamics. Therefore, in the following we focus on the nutation and precession control, for which a hybrid MPC scheme will be devised.

We introduce the state vector $x(t)=\left[\tilde{\Theta}_{x}(t), \tilde{\Psi}_{x}(t)\right.$, $\left.\omega_{y}(t), \omega_{z}(t)\right]^{\prime}$ and the input vector $u(t)=\left[\tau_{y}(t), \tau_{z}(t)\right]^{\prime}$. The linear time-invariant system (4) is discretized using sampling time $T_{s}=0.5 \mathrm{~s}$ and assuming that the digital-to-analog coupling is realized by means of a zero-order hold element, i.e., $u(t)=u(k)$ for $t \in\left[k T_{s},(k+1) T_{s}\right)$ for all $k \in \mathbb{N}$. This gives rise to the following discrete-time linear time invariant system

$$
x(k+1)=A x(k)+B u(k),
$$

which will be used as the plant model in the formulation of the MPC problem in Section III.

\section{B. Minimum Impulse Thrusters}

The torques $\tau_{y}$ and $\tau_{z}$ exerted by the thrusters on the $y$ and $z$ axes, respectively, are subject to a Minimum Impulse Bit (MIB), meaning that, once the thrusters are turned on, they cannot be turned off immediately. The minimum time interval for which the thrusters must stay on implies a minimum exerted torque on the corresponding axis. This can be modelled by non-convex constraints of the form $u(k) \in \mathcal{U}$, with

$$
\mathcal{U}=\left[-u_{\max }, u_{\min }\right] \cup\{0\} \cup\left[u_{\min }, u_{\max }\right]
$$

where $u_{\text {min }} \in \mathbb{R}^{2}$ denotes the minimum impulse bit and $u_{\text {max }} \in \mathbb{R}^{2}$ denotes the maximum torque that can be provided in a sampling interval on each axis. In order to 
translate this constraint to a computationally tractable form, we consider the convex constraints

$$
-u_{\max } \leq u(k) \leq u_{\max },
$$

introduce the binary vector $\delta(k)=$ $\left[\delta_{11}(k), \delta_{12}(k), \delta_{21}(k), \delta_{22}(k)\right]^{\prime}, \quad$ and establish the correspondence

$$
\begin{aligned}
& \delta_{i 1}(k)=\left[u_{i}(k) \leq-u_{\min , i}\right], \text { for } i \in\{1,2\}, \\
& \delta_{i 2}(k)=\left[u_{i}(k) \geq u_{\min , i}\right], \text { for } i \in\{1,2\} .
\end{aligned}
$$

Notice that whenever $\delta_{i 1}=1$ or $\delta_{i 2}=1$, the control action $u$ is outside the interval $\left(-u_{\min }, u_{\min }\right)$, so in light of (7) it can be applied to the system. We define the following propositional logic constraints on the auxiliary continuous variables $z(k) \in \mathbb{R}^{2}$ :

$$
\left.z_{i}(k)=\left[\delta_{i 1}(k) \vee \delta_{i 2}(k)\right] \cdot u_{i}(k)\right), \forall i \in\{1,2\}
$$

where " $\vee$ " stands for the logical disjunction (OR) operator. Additionally, we introduce the auxiliary continuous variables $z_{\text {act }}(k) \in \mathbb{R}^{2}$ to trace whether at every time instant a thruster activation takes place:

$$
z_{\text {act }, i}(k)=\left[\delta_{i 1}(k) \vee \delta_{i 2}(k)\right], i \in\{1,2\} .
$$

By (7), (8) and (9) we have $z(k) \in \mathcal{U}$. Hence, the system dynamics subject to the thrusters constraints can be described by the linear discrete-time model where the continuous variable $u$ has been replaced by the hydrid variable $z$ where the relationship between these is given by (9).

$$
\begin{aligned}
x(k+1) & =A x(k)+B z(k) \\
\gamma(k+1) & =\gamma(k)+\left[\begin{array}{ll}
1 & 1
\end{array}\right] z_{a c t}(k),
\end{aligned}
$$

where the additional state variable $\gamma(k) \in \mathbb{R}$, namely the activation count, stands for the number of thrusters activations and, if necessary, can be bounded by the number of maximum activations allowed $\gamma_{\max }$ according to:

$$
\gamma(k) \leq \gamma_{\max }
$$

This constraint is likely to become active only if the prediction horizon is long enough to foresee the exhaustion of available actuations or $\gamma(k)$ is close to $\gamma_{\max }$.

\section{MPC FORMULATION}

MPC is an optimization-based control strategy: at each sampling instant a performance index is optimized using a discrete-time dynamical model of the system subject to constraints, yielding a sequence of control moves over a future time window. Then, the first element of this sequence is the control action applied to the system, while the other elements are discarded.

Let $\pi_{N} \triangleq\left\{u(k), z(k), z_{\text {act }}(k), \delta(k)\right\}_{k=0}^{N-1}$ be the set of optimization variables, $x_{0}$ the current state, and $\gamma_{0}$ the total number of thrusters activations up to time $k$. We propose the following hybrid MPC problem:

$$
\begin{array}{ll}
\min _{\pi_{N}} & V\left(\pi_{N}, x_{0}, \gamma_{0}\right) \\
\text { s.t. } & x(0)=x_{0}, \gamma(0)=\gamma_{0}, \\
& \text { Constraints (7)-(12), for } k \in \mathbb{N}_{\left[0, N_{u}\right]}, \\
& \delta\left(N_{u}+k\right)=0, \text { for } k \in \mathbb{N}_{\left[0, N-N_{u}-1\right]},
\end{array}
$$

where $N \geq 1$ defines the prediction horizon, i.e., the size of the future time window for which the system evolution is predicted, and $N_{u} \leq N$ defines the control horizon, i.e., the number of time steps for which the control moves can be optimized. A control horizon which is small compared to the prediction horizon is typically employed to reduce the complexity of the resulting optimization problem. In the proposed formulation (13), the input is assumed to satisfy all constraints given by equations (7)-(12) for all $k \in \mathbb{N}_{\left[0, N_{u}\right]}$, and no activation is allowed after the control horizon as in $(13 \mathrm{~d})$.

Due to the upper-stage rotational dynamics, which is very slow compared to the sampling time $T_{s}=0.5 \mathrm{~s}$, a relatively long prediction horizon is required to allow MPC to predict far enough in the future. On the other hand, a short control horizon is needed to limit the number of optimization variables, so as to keep the complexity of (13) reasonably low.

The term $V$ in (13a) is the performance index, defined as

$$
V\left(\pi_{N}, x_{0}, \gamma_{0}\right) \triangleq V_{N}(x(N), \gamma(N))+\sum_{k=0}^{N-1} \ell(x(k), z(k)),
$$

where $\ell$ is the stage cost, $\ell(x, z) \triangleq\|Q x\|_{p}+\|R z\|_{p}$ and $V_{N}$ is the terminal cost, $V_{N}(x(N), \gamma(N)) \triangleq\left\|Q_{N} x(N)\right\|_{p}+$ $\rho\left(\gamma(N)-\gamma_{0}\right)$, and $Q, R, Q_{N}$, and $\rho$ are weight matrices used to obtain the desired trade-off between tracking performance and thrusters usage, and the notation $\|\cdot\|_{p}$ is either the $\infty$ norm $\left(\|\cdot\|_{\infty}, p=\infty\right)$ or the squared Euclidean norm $\left(\|\cdot\|_{2}^{2}\right.$, $p=2$ ). The choice $p=\infty$ leads to the formulation of a mixed integer linear programming problem (MILP), while $p=2$ leads to a mixed integer quadratic programming problem (MIQP); here, we use $p=\infty$ to lower the computational complexity of (13). The term $\rho\left(\gamma(N)-\gamma_{0}\right)$ in $V_{N}$ is used to penalize the total number of thrusters activation along the prediction horizon to eventually lead to a low-activation attitute control of the spacecraft.

Recently Frick et al. [17] proposed the use of a standard branch-and-bound algorithm combined with a fast embedded interior point solver and certain heuristics that considerably speed-up the solution of such hybrid MPC optimization problems and yield near-optimal solutions. This allows for an efficient on-chip implementation of the proposed hybrid model predictive controller.

The purpose of control problem (13) is to drive the pitch and yaw error angles in an orbit close to zero, while satisying the MIB constraints (7)-(10), avoiding thrusters activation as much as possible, and respecting the constraint (12) on the total number of actuations. As mentioned above, the 
desired compromise between these contrasting objectives is determined by the choice of the weight parameters $Q, R, Q_{N}$ and $\rho$. Unless otherwise stated, in the following we set $R=0$ since we make use of the weight coefficient $\rho$ to penalize the total number of activations. In general, having nonzero $R$ can be useful to reduce the consumption of propellant needed to actuate the thrusters, if relevant. With $R=0$, inputs are still indirectly penalized through the actuation count variable $\gamma$.

The MPC control action is computed in a receding horizon fashion: The optimization problem (13) is solved at every sampling time instant and returns an optimizer, that is a sequence $\pi_{N}^{\star}(x, \gamma)=$ $\left\{\left(u^{\star}(k ; x, \gamma), z^{\star}(k ; x, \gamma), z_{\text {act }}^{\star}(k ; x, \gamma), \delta^{\star}(k ; x, \gamma)\right)\right\}_{k=0}^{N-1}$.

Then, the first value of $z^{\star}$, namely $z^{\star}(0)$, is used as the control action and it is applied to the system. The control action is hence given by the feedback law

$$
\kappa(x, \gamma)=z^{\star}(0 ; x, \gamma) .
$$

The MPC controller commands admissible torques to the thrusters which will be activated for a certain time between $t_{\min }$ and $T_{s}$, where $t_{\min }$ is the minimum time for which the thruster can remain open (and corresponds to a $u_{\min }$ torque) and $T_{s}$ is the sampling time. The proposed methodology grants a clear advantage over other approaches to attitude control that only provide on/off commands to the thrusters, such as the one in [18]. In fact, with the proposed approach the sampling time $T_{s}$ can be much larger than the minimum impulse time $t_{\min }$, and as consequence the MPC controller can have a greater foresight of the system evolution at a much lower computational cost.

\section{Upper Stage Attitude Control DURing LONG COASTING PERIODS}

Here we demonstrate how the proposed control approach performs based on the nonlinear kinematic model (2), which was built based on real data.

The state variables of the upper stage are measured by a high-precision Inertial Measurement Unit (IMU) which measures the translational acceleration and the angular rates of the spacecraft using three accelerometers and three laser gyroscopes.

\section{A. Control under nominal conditions}

In this section the performance of the proposed hybrid MPC are tested in nominal conditions, that is, in the absence of external disturbances and neglecting the effects of fuel sloshing. In the considered simulation settings, the moments of inertia are $J_{x x}=77.2 \cdot 10^{3} \mathrm{~kg} \mathrm{~m}{ }^{2}, J_{y y}=$ $J_{z z}=93.8 \cdot 10^{4} \mathrm{~kg} \mathrm{~m}^{2}$, the maximum torque bounds are $u_{\max }=[5000,5000]^{T} \mathrm{Nm}$, the minimum torque bounds due to MIB are $u_{\min }=[200,200]^{T} \mathrm{Nm}$, the roll rate setpoint is $\omega_{x}^{r}=5 \frac{\pi}{180} \mathrm{rad}$, and the initial angular rates are $\omega_{x}(0)=-1 \frac{\pi}{180} \mathrm{rad} / \mathrm{s}, \omega_{y}(0)=\omega_{z}(0)=0.1 \frac{\pi}{180} \mathrm{rad} / \mathrm{s}$. The weight matrices were set to $Q=Q_{N}=\operatorname{diag}(1,1,0,0)$, $R=0$ and $\rho=0.1$. A good balance between computational complexity and performance of the controller was achieved for $N=120$ and $N_{u}=15$.
TABLE I: Simulation results over an interval $T_{\text {sim }}=300 \mathrm{~s}$ in nominal conditions.

\begin{tabular}{|l|r|r|r|r|c|}
\hline & \multicolumn{4}{|c|}{ Thruster activations } & \multirow{2}{*}{$J_{r}$} \\
\cline { 2 - 5 } & $x$-axis & $y$-axis & $z$-axis & total & \\
\hline \hline PD controller & 9 & 7 & 34 & 50 & 0.4741 \\
LQ controller & 9 & 21 & 36 & 66 & 0.3858 \\
Hybrid MPC & 9 & 4 & 5 & 18 & 0.0811 \\
\hline
\end{tabular}

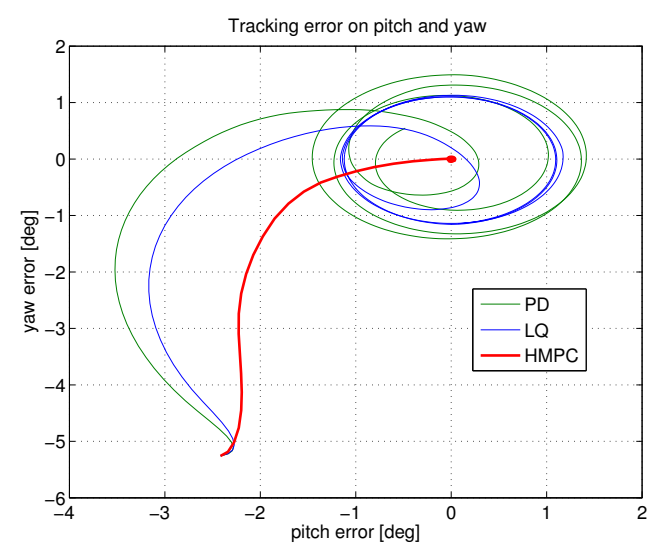

Fig. 2: Trajectories of the pitch and yaw error angles $\tilde{\Theta}_{x}, \tilde{\Psi}_{x}$ for the proposed hybrid MPC controller and comparison with the PD and an LQR controller of [3].

The hybrid MPC is compared with two controllers based on $\mathrm{PD}$ and LQR, respectively, as designed in [3] whose control actions are saturated by the following mapping:

$$
\operatorname{sat}(v)=\left\{\begin{array}{l}
0, \text { if }|v| \leq u_{\min } \\
\frac{v}{|v|} \min \left\{v^{2} /|v|, u_{\max }\right\}, \text { otherwise }
\end{array}\right.
$$

The main difference between those controllers and MPC is that they do not take explicitly into account the MIB effect or other constraints on thrusters actuation. The results of a simulation over an interval of $T_{\mathrm{sim}}=300 \mathrm{~s}$ are summarized in Table I, where the number of thrusters activations produced by different controllers is shown, together with a performance index based on the pitch and yaw tracking error:

$$
J_{r}=\sum_{k=0}^{T_{\mathrm{sim}}}\left(\tilde{\Theta}_{x}^{2}(k)+\tilde{\Psi}_{x}^{2}(k)\right) .
$$

For the sake of completeness, in Table I we also report the number of activations on the $x$-axis, that is achieved by a simple linear gain controller for either MPC, PD, and LQR controllers. Trajectories of pitch and yaw error angles $\tilde{\Theta}_{x}$, $\tilde{\Psi}_{x}$ are shown in Figure 2, while thrusters' commands for the three controllers are shown in Figure 3.

We observe that the proposed hybrid MPC controller greatly outperforms the other two controllers (PD and LQR), both in terms of number of thrusters activations and of angles error. This is mainly due to the capability of MPC to model the actuators' nonlinearity (6) and to explicitly penalize the activations of the thrusters in the cost function. At the same time, as shown in Figure 4, the roll rate equilibrates fast at the desired set-point which justifies our assumption to consider 

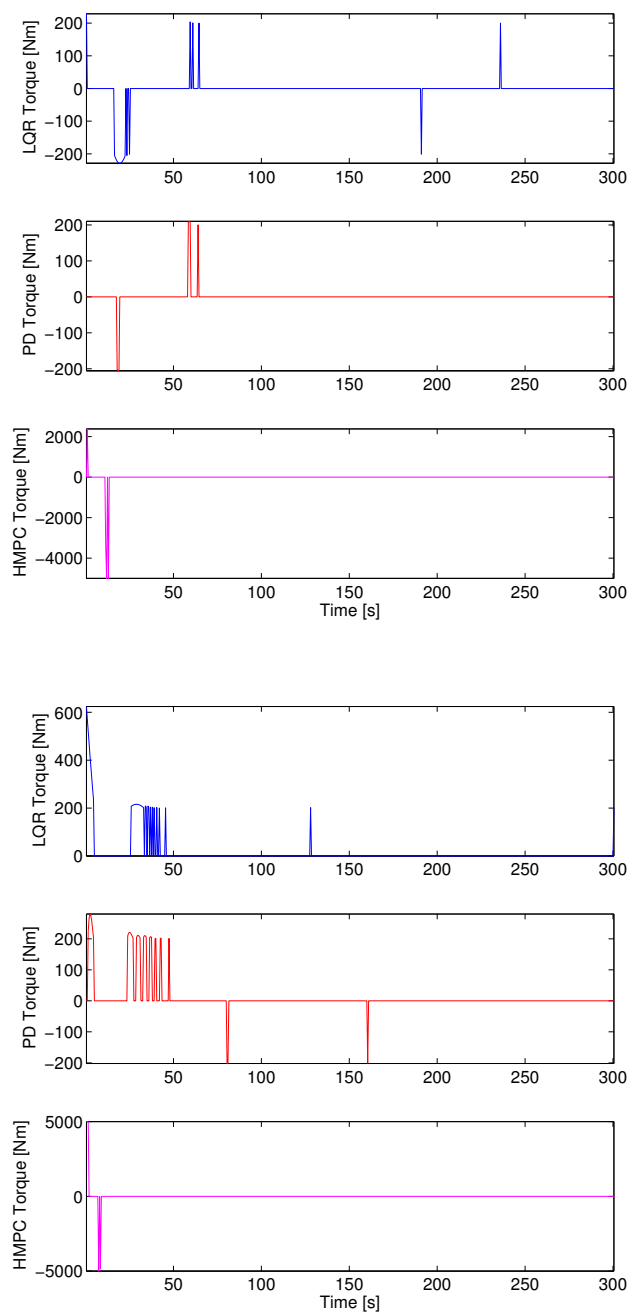

Fig. 3: Torques applied on the $y$-axis (up) and the $z$-axis (down) by the LQR, the PD and the hybrid MPC controller.

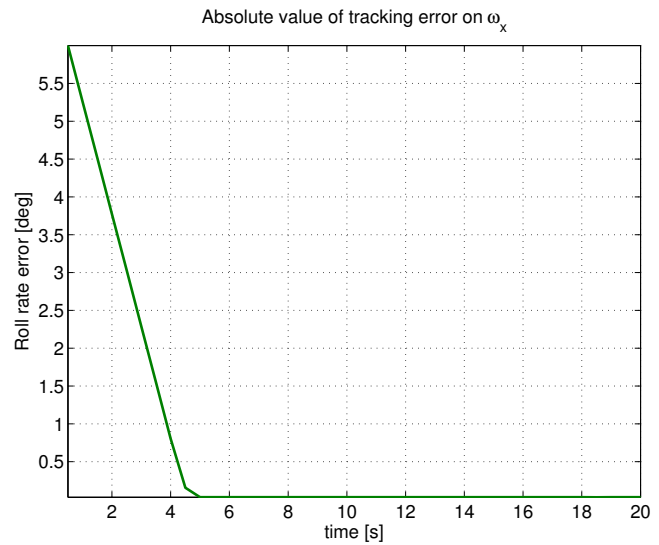

Fig. 4: The roll rate converges fast to the desired set-point of $5 \mathrm{deg} / \mathrm{s}$.

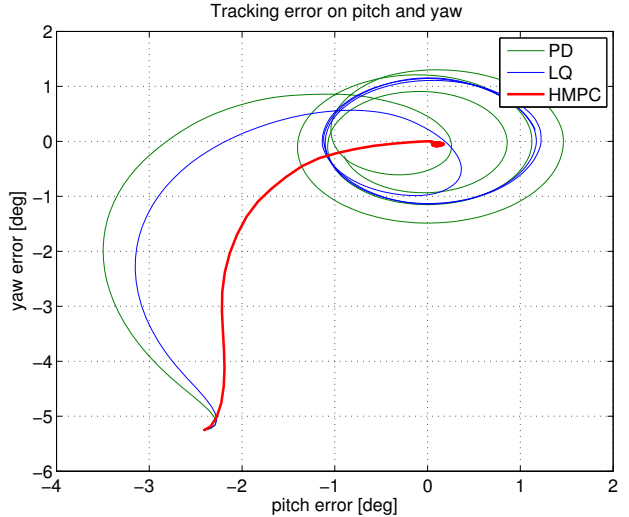

Fig. 5: Trajectories of the pitch and yaw error angles $\tilde{\Theta}_{x}, \tilde{\Psi}_{x}$ for the proposed hybrid MPC controller and comparison with the PD and an LQR controller of [3] in presence of a constant torque disturbance.

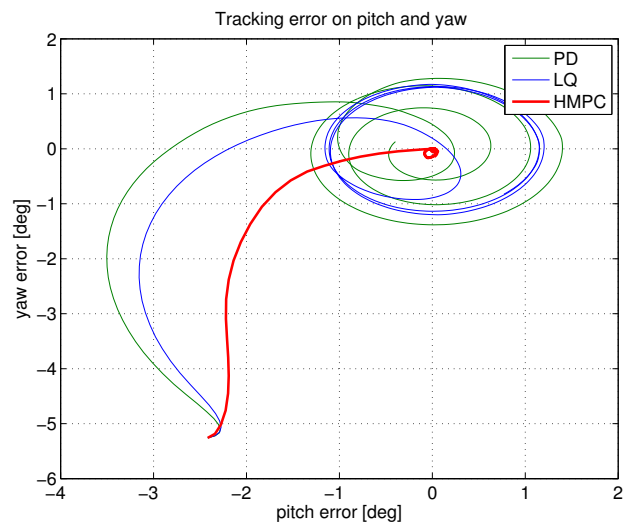

Fig. 6: Trajectories of the pitch and yaw error angles $\tilde{\Theta}_{x}, \tilde{\Psi}_{x}$ for the proposed hybrid MPC controller and comparison with the PD and an LQR controller of [3] in the presence of a sinusoidal torque disturbance.

it constant and equal to its equilibrium value for the design of the MPC controller.

\section{B. Control in presence of unmodelled disturbances}

Here we test the proposed hybrid MPC for robustness in presence of unmodelled torque disturbances. In particular, we examine two scenarios: constant torque disturbances on the $y$ - and $z$-axis of magnitude $6 \mathrm{Nm}$ and a sinusoidal torque disturbance between 0.5 and $6 \mathrm{Nm}$ at $0.2 \mathrm{~Hz}$. Such torque disturbances correspond to worst-case scenarios in regard to actuation imprecisions.

TABLE II: Comparative analysis of the attitude controllers in the presence of a constant torque of $6 \mathrm{Nm}$ on the $y$-axis and another $6 \mathrm{Nm}$ on the $z$-axis.

\begin{tabular}{|l|r|r|r|r|c|}
\hline & \multicolumn{4}{|c|}{ Thruster activations } & \multirow{2}{*}{$J_{r}$} \\
\cline { 2 - 5 } & $x$-axis & $y$-axis & $z$-axis & total & \\
\hline \hline PD controller & 9 & 11 & 30 & 50 & 0.4454 \\
LQ controller & 9 & 26 & 40 & 75 & 0.3878 \\
Hybrid MPC & 9 & 16 & 5 & 30 & 0.0880 \\
\hline
\end{tabular}


TABLE III: Comparative analysis of the attitude controllers in presence of sinusoidal torque disturbances along the $y$ and $z$ axes.

\begin{tabular}{|l|r|r|r|r|c|}
\hline & \multicolumn{4}{|c|}{ Thruster activations } & \multirow{2}{*}{$J_{r}$} \\
\cline { 2 - 5 } & $x$-axis & $y$-axis & $z$-axis & total & \\
\hline \hline PD controller & 9 & 10 & 31 & 50 & 0.4024 \\
LQ controller & 9 & 24 & 37 & 70 & 0.3899 \\
Hybrid MPC & 9 & 9 & 8 & 26 & 0.0869 \\
\hline
\end{tabular}

As presented in Tables II and III, the presence of a disturbance entails an increase in the number of actuations needed to control the attitude of the upper stage. Still, the proposed hybrid MPC controller outperforms the PD and LQR controllers significantly.

\section{Computational Complexity}

The use of a control horizon that is lower than the prediction horizon in our hybrid MPC formulation enables us to control the complexity of the mixed-iteger optimization problem we need to solve. Using Gurobi as the MILP solver, the hybrid MPC optimization problem was solved in $0.21 \mathrm{~s}$ per sampling step on average, with a standard deviation of $0.26 \mathrm{~s}$, on a $2.2 \mathrm{GHz}$ Intel Core $i 7$.

\section{CONCLUSIONS AND FUTURE WORK}

In this paper we modelled the minimum impulse bit of the thrusters in a hybrid systems framework and devised a hybrid MPC controller that aims at correcting the yaw and pitch errors while minimizing the number of thruster actuation.

We demonstrated that the closed-loop trajectories of the controlled system are closer to the control objective than the ones generated by conventional control approaches such as PD or LQR. Additionally, we showed that the proposed MPC scheme leads to a significant reduction of the number of thruster activations compared to those obtained using PD and LQR controllers.

This paper offers a proof-of-concept in regard to the use of hybrid MPC for the attitude control of upper stage launchers taking into account the MIB effect. For an embedded implementation of the proposed control approach, one needs to develop an efficient algorithm for the online solution of (13). Even if the implementation of a hybrid MPC controller as a flight algorithm is rejected (for reasons of limited computing resources or verification problems), our work gives an indication of the optimal solution against which other approaches can be benchmarked.

Although stability properties of the controlled system are not theoretically proven in the paper, exhaustive simulations have shown that the state of the controlled system is steered to a small neighbourhood of the origin with significantly fewer actuations compared to PD and LQR controllers typically employed for attitude control.

Future work will focus on the design of a hybrid MPC controller that takes into account the effect of the sloshing of fuel in the tanks of the spacecraft, resulting in additional torque disturbances and possibly off-diagonal inertias [19], and exploits reduced-order models in order to limit the complexity of the resulting controller [20]. In addition, efficient
MPC formulations that trade optimality of the control action for lower CPU requirements will be investigated, in order to address embedded implementation.

\section{REFERENCES}

[1] J.-Y. Wen and K. Kreutz-Delgado, "The attitude control problem," Automatic Control, IEEE Transactions on, vol. 36, pp. 1148-1162, Oct 1991.

[2] L. Wang, Z. Li, and B. Wang, "Robust satellite attitude control," in Advances in Computer Science and Information Engineering (D. Jin and S. Lin, eds.), vol. 168 of Advances in Intelligent and Soft Computing, pp. 649-654, Springer Berlin Heidelberg, 2012.

[3] A. Kater, "Attitude Control of Upper Stage Launcher During Long Coasting Period," Master's thesis, Lehrstuhk f ur Automatisierungsund Regelungstechnik, Christian-Albrechts-University Kiel, Germany, 2013.

[4] R. Huftalen, J. Parker, A. Platt, and G. Yankura, "Minimum impulse thruster valve design and development," in Proceedings of the 4th International Spacecraft Propulsion Conference (ESA SP-555), pp. 6878, June 2004.

[5] J. B. Rawlings and D. Q. Mayne, Model Predictive Control: Theory and Design. Nob Hill Publishing, 2009.

[6] V. Manikonda, P. Arambel, M. Gopinathan, R. Mehra, and F. Hadaegh, "A model predictive control-based approach for spacecraft formation keeping and attitude control," in American Control Conference, 1999. Proceedings of the 1999, vol. 6, pp. 4258-4262, 1999.

[7] M. Vieira, R. Galvao, and K. Heinz Kienitz, "Attitude stabilization with actuators subject to switching-time constraints using explicit MPC," in 2011 IEEE Aerospace Conference, pp. 1-8, March 2011.

[8] O. Hegrenas, J. Gravdahl, and P. Tondel, "Attitude control by means of explicit model predictive control, via multi-parametric quadratic programming," in American Control Conference, 2005. Proceedings of the 2005, pp. 901-906 vol. 2, June 2005.

[9] O. Hegrenas, J. Gravdahl, and P. Tondel, "Spacecraft attitude control using explicit model predictive control," Automatica, vol. 41, no. 12, pp. 2107 - 2114, 2005.

[10] B. Xiao, Q. Hu, and P. Shi, "Attitude stabilization of spacecrafts under actuator saturation and partial loss of control effectiveness," Control Systems Technology, IEEE Transactions on, vol. 21, pp. 2251-2263, Nov 2013.

[11] A. Bemporad and M. Morari, "Control of systems integrating logic, dynamics, and constraints," Automatica, vol. 35, no. 3, pp. 407 - 427, 1999.

[12] W. Heemels, B. de Schutter, and A. Bemporad, "Equivalence of hybrid dynamical models," Automatica, vol. 37, no. 7, pp. 1085-1091, 2001.

[13] N. Giorgetti, A. Bemporad, H. Tseng, and D. Hrovat, "Hybrid model predictive control application towards optimal semi-active suspension," Int. J. Control, vol. 79, no. 5, pp. 521-533, 2006.

[14] F. Borrelli, A. Bemporad, M. Fodor, and D. Hrovat, "An MPC/hybrid system approach to traction control," IEEE Trans. Contr. Systems Technology, vol. 14, no. 3, pp. 541-552, 2006.

[15] D. Bernardini, S. Di Cairano, A. Bemporad, and H. Tseng, "Driveby-wire vehicle stabilization and yaw regulation: A hybrid model predictive control design," in Proc. 48th IEEE Conf. on Decision and Control, (Shanghai, China), pp. 7621-7626, 2009.

[16] S. Di Cairano, D. Bernardini, H. Tseng, and A. Bemporad, "Vehicle yaw stability control by coordinated active front steering and differential braking in the tire sideslip angles domain," IEEE Trans. Contr. Systems Technology, vol. 1, no. 4, pp. 1236-1248, 2013.

[17] D. Frick, A. Domahidi, and M. Morari, "Embedded Optimization for Mixed Logical Dynamical Systems," Computers \& Chemical Engineering, July 2014. to appear.

[18] M. Leomanni, A. Garulli, A. Giannitrapani, and F. Scortecci, "An mpc-based attitude control system for all-electric spacecraft with on/off actuators," in Proc. 52th IEEE Conf. on Decision and Control, (Florence, Italy), pp. 4853-4858, 2013.

[19] A. E. P. Veldman, J. Gerrits, R. Luppes, J. A. Helder, and J. P. B. Vreeburg, "The numerical simulation of liquid sloshing on board spacecraft," J. Comput. Physics, vol. 224, no. 1, pp. 82-99, 2007.

[20] P. Sopasakis, D. Bernardini, and A. Bemporad, "Constrained model predictive control based on reduced-order models," in 52nd IEEE Conference on Decision and Control, IEEE, (Firenze, Italy), pp. 70717076, Dec. 2013. 